\title{
Caracterización dinámica de escaleras mecánicas mediante modelos simulación de sistemas multicuerpo
}

\section{Dynamic characterization of escalators using multibody simulation models}

Juan David Cano-Moreno
Profesor asociado, Universidad Politécnica de Madrid, Departamento de Ingeniería Mecánica,
Química y Diseño Industrial.
España.
juandavid.cano@upm.es
ORGID: https://orcid.org/0000-0002-4868-7927
José María Cabanellas Becerra
Profesor titular, Universidad Politécnica de Madrid, Departamento de Ingeniería Mecánica.
España.
ORCID: https:/ / orcid.org/0000-0001-8443-214X

Recepción: 01/02/2018. Aceptación: 29/05/2018. Publicación: 14/06/2018

Citación sugerida:

Cano-Moreno, J. D. y Cabanellas Becerra, J. M. (2018). Caracterización dinámica de escaleras mecánicas mediante modelos simulación de sistemas multicuerpo. 3C Tecnología: Glosas de innovación aplicadas a la pyme, 7(2), 82-103. DOI: http://dx.doi.org/10.177993/3ctecno.2018.v7n2e26.82-103/ 


\section{RESUMEN}

En este trabajo se propone usar los modelos de simulación dinámica para mejorar los diseños ya existentes e innovar con nuevos diseños, en un sector en previsible declive.

La problemática de los modelos dinámicos de escaleras es amplia, empezando porque es un diseño robusto y probado en los más de 100 años que lleva casi sin variaciones. En segundo lugar, es un sistema multicuerpo articulado formado por un número muy elevado de cuerpos rígidos, lo que supone un esfuerzo de modelización enorme sin las herramientas adecuadas.

La metodología paramétrica escalable utilizada permite automatizar las tareas más costosas para producir un modelo de simulación flexible y adaptado a las características particulares de la escalera en estudio.

Como paso necesario para establecer la metodología está su validación con un sistema real instrumentado. El contraste de resultados con el modelo real instrumentado valida el modelo de simulación e inferir de él características dinámicas para otros modelos simulados.

El tipo de trabajo presentado aquí funciona como cimiento para el desarrollo de modelos cada vez más completos, como ya ha sucedido en otras áreas de simulación.

\section{ABSTRACT}

In this work, it is proposed to use dynamic simulation models to improve existing and innovative designs with new designs, in a sector in foreseeable decline.

The problematic of the dynamic models of escalators is wide, beginning because it is a robust and proven design in the more than 100 years that it has with almost no variations. Secondly, it is an articulated multibody system formed by a very high number of rigid bodies, which implies a huge modeling effort without the adequate tools.

The scalable parametric methodology used to automate the most expensive tasks to produce a flexible simulation model adapted to the particular characteristics of the escalator under study.

As a necessary step to establish the methodology is its validation with a real instrumented system. The contrast of 
results with the real instrumented model validates the simulation model and infers its dynamic characteristics for other simulated models.

The type of work presented here works as a foundation for the development of increasingly complete models, as has already happened in other areas of simulation.

\section{PALABRAS CLAVE}

Escalera mecánica, Validación, SIMPACK, Dinámica, Multicuerpo, Transporte vertical.

\section{KEY WORDS}

Escalator, Validation, Dynamic, Multibody, Vertical transport. 


\section{INTRODUCCIÓN}

El diseño básico de la escalera mecánica no ha variado sustancialmente desde hace más de 100 años [1]. Este estudio muestra que en el sector de escaleras y pasillos mecánicos hay muchas ideas patentadas que no han conseguido comercializarse. Esta poca permeabilidad de las invenciones al mercado (innovación) supone un reto para los nuevos diseños.

Sin embargo, los problemas de seguridad y de competitividad están haciendo presión para que este sector evolucione hacia nuevos paradigmas de diseño.

Para acelerar la etapa el análisis y mejora de los diseños actuales o nuevos de escaleras mecánicas, se ha desarrollado una metodología que permite implementar modelos de simulación dinámica de escaleras mecánicas y pasillos móviles, permitiendo así ahorrar tiempo y dinero frente al uso de prototipos reales.

La simulación dinámica de sistemas multicuerpo constituye una herramienta cada vez más empleada en las fases de diseño y análisis de un sistemas mecánicos [2], [3].

En la literatura se encuentran escasas referencias simulación de escaleras. Una en Corea, Yi Sug Kwon, perteneciente a la empresa OTIS-LG Elevator, del que destaca un modelo completo de escalera mecánica convencional implementado en DADS [4]. Tiene además Kwon un modelo de pasamanos[5], en el que se reflejan resultados obtenidos experimentalmente. El objetivo de este modelo es de servir como guía de diseño para este subsistema de la escalera mecánica.
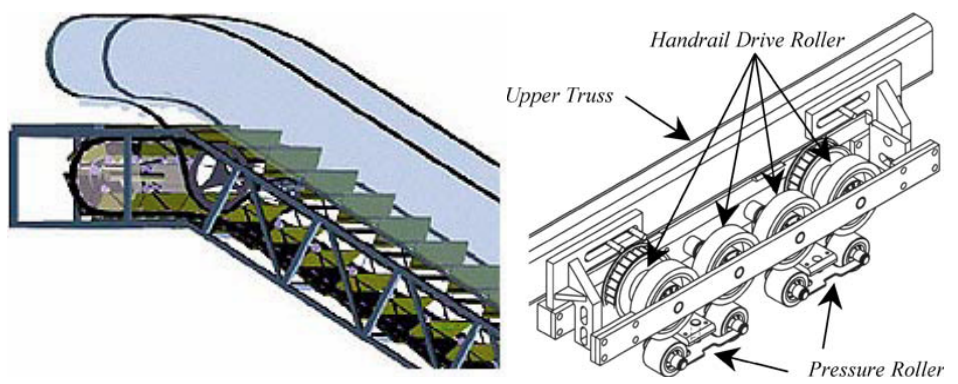

Gráfico 1. Modelos simulados por Yi Sug Kwon. 
Además, destaca la publicación de Kwon de un modelo dinámico de un pasamanos [5] , en el que se reflejan resultados obtenidos experimentalmente. El objetivo de este modelo es de servir como guía de diseño para este subsistema de la escalera mecánica.

Una segunda referencia se encuentra en Japón. Isotani Hitoshi (Hitachi) ha publicado un artículo sobre un modelo dinámico completo de escalera mecánica [6].

La forma de actuar que se propone en este trabajo puede considerarse una buena práctica a la hora de catalizar la innovación [7], [8]en este sector que se está estancando.

\section{FUNCIONAMIENTO DE UNA ESCALERA MECÁNICA}

Se puede considerar que una escalera mecánica está formada por una cadena de peldaños que se mueven a lo largo de un bucle continuo siguiendo una forma específica determinada por unas guías.

En el Gráfico 2 se muestran las principales partes y zonas de una escalera mecánica convencional con la nomenclatura que se empleará. De cara al usuario solo quedan visibles los peldaños de las zonas superiores situadas entre las zonas de embarque indicadas.

Una escalera mecánica está formada por una cadena de peldaños que se mueven a lo largo de un bucle continuo siguiendo una forma específica determinada por unas guías. 


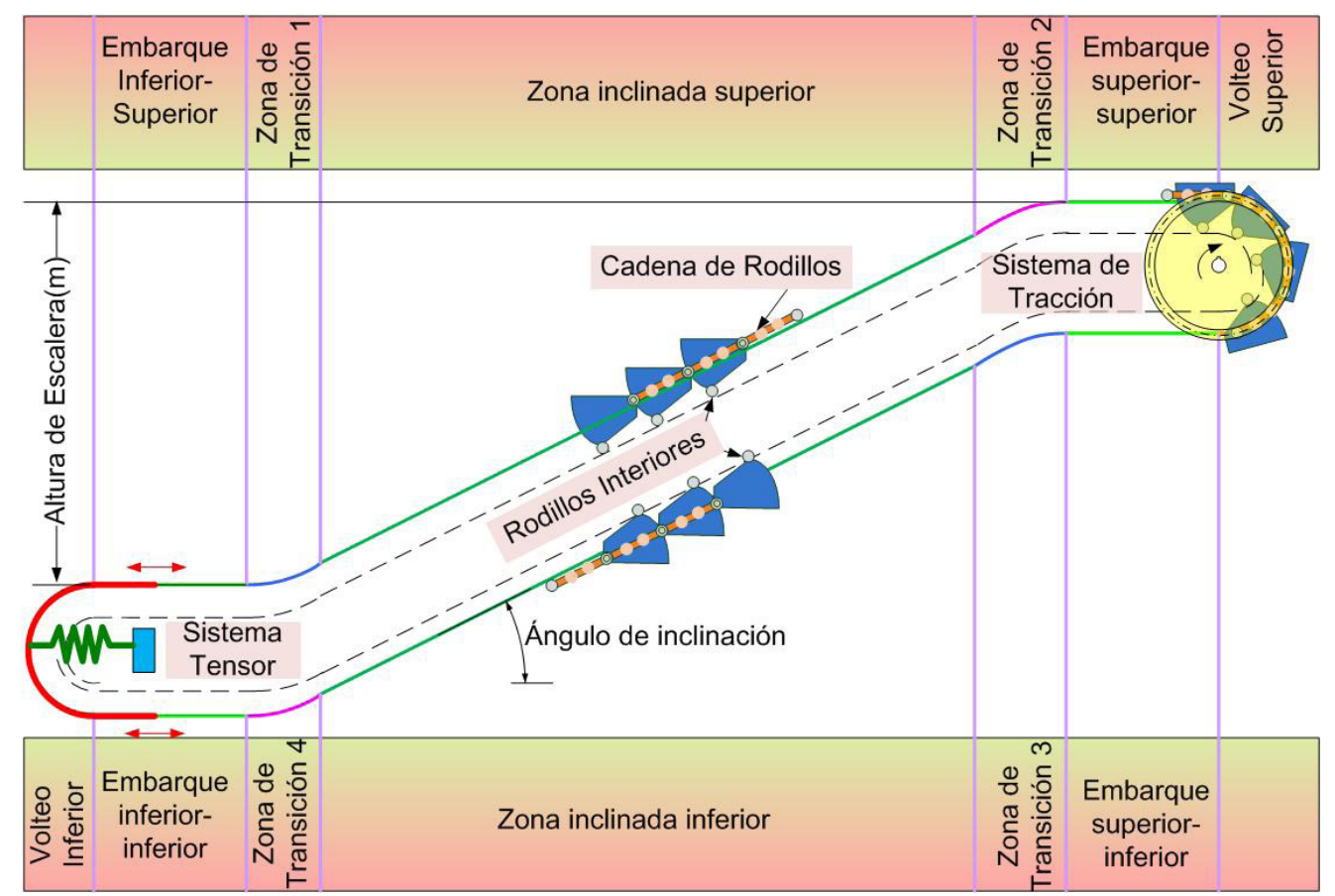

Gráfico 2. Principales partes de una escalera mecánica convencional.

\section{SUBSISTEMAS A MODELAR}

En una escalera completa hay subsistemas que directamente afectan a su dinámica, peldaños, cadenas, guías, tensor y tracción, y que van a ser los objetos directos de este estudio. Otros subsistemas afectan indirectamente a su dinámica, bastidor, pasamanos, motor y sistema de control. De estos sistemas se modelizará el motor conjuntamente con la parte del control que se encarga de la regulación de la velocidad. Una tercera serie de subsistemas como la iluminación, frenado, seguridad, balaustrada y otros, no afectan a la dinámica de funcionamiento salvo en casos particulares y por lo tanto no se modelan. 


\section{CADENAS Y PELDAÑOS}

Cada peldaño está unido a sendas cadenas, en los denominados rodillos exteriores, pero también poseen dos rodillos interiores que ruedan libremente por sus guías correspondientes. De este modo, cada peldaño puede definirse como un carro apoyado en cuatro rodillos. Las cadenas empleadas en estos sistemas de transporte vertical suelen poseer dos mallas de cadena entre cada articulación, y un rodillo por articulación, Gráfico 3. Su modelado requiere permitir el giro relativo entre eslabones y entre los rodillos de dicha cadena y los eslabones. También es necesario modelar el contacto rodilloguía.
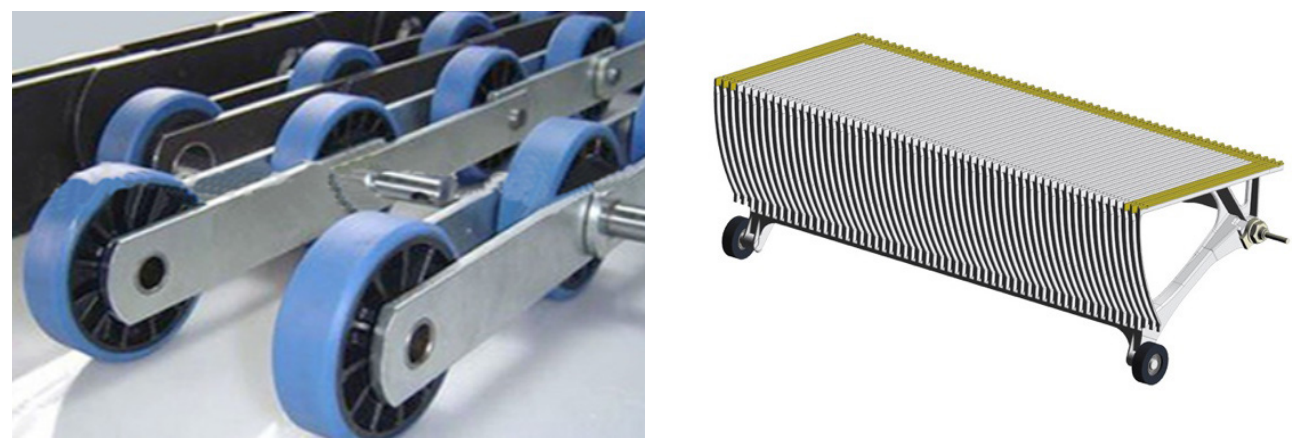

Gráfico 3. Cadena de rodillos y peldaño.

Los peldaños son cuerpos realizados de una sola pieza, con 4 articulaciones, dos a cada lado. En cada lado está ligado a dos rodillos, uno perteneciente a la cadena de rodillos y, el otro, gira libre respecto del peldaño y apoya en su correspondiente guía.

\section{SISTEMA TENSOR}

Se emplean como elemento tensor dos muelles, uno por cadena, situado en la cabecera inferior sobre guías deslizantes. La colocación inferior es debida a que es dónde menor tensión de cadena se produce. Con el tensor se asegura que la cadena está siempre trabajando a tracción, evitando de esta forma los pandeos y pérdidas de guía que se producen en la compresión de un sistema 
articulado. La capacidad deslizante permite absorber los cambios geométricos del fenómeno de poligonalización, que se produce por la aproximación de una curva por polígonos de lado igual a la longitud de eslabón.
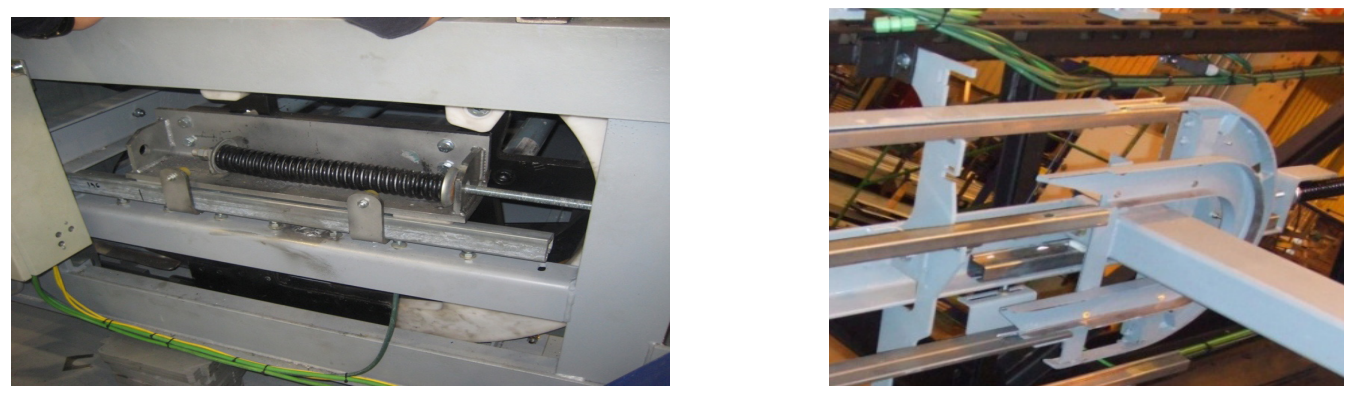

Gráfico 4. Muelle-y guías de la estación tensora.

\section{SISTEMA DE ACCIONAMIENTO}

Se denomina sistema de accionamiento a aquel que transmite el movimiento a los peldaños. El sistema lo forman las cadenas de rodillos para el movimiento longitudinal a lo largo del recorrido y las guías interiores que actuando sobre los dos rodillos libres del peldaño hacen girar el peldaño alrededor de los rodillos fijos con las cadenas. Mediante este giro se consigue que el peldaño este en la posición correcta en el recorrido, horizontal en con los pasajeros y recogido en el resto.

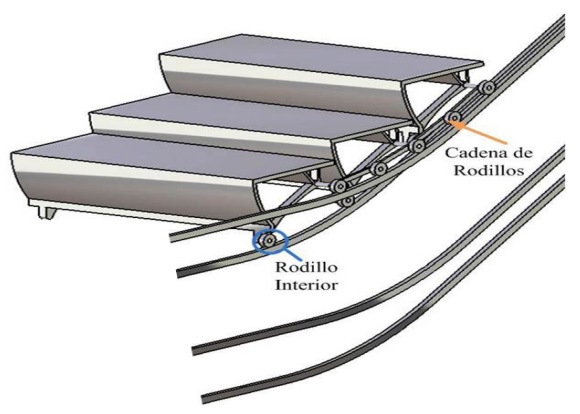

Gráfico 5. Peldaños de una escalera mecánica. 


\subsection{SISTEMA DE TRACCIÓN}

Este sistema se refiere al sistema que mueve la cadena de rodillos. Generalmente consiste en dos ruedas dentadas que engranan en los rodillos de las cadenas. Las ruedas están unidas rígidamente por un eje y se sitúan en la cabecera superior. Existen numerosas patentes y algunas escaleras en la realidad que funcionan con sistemas de tracción lineales situados en la zona inclinada. Estas ruedas a su vez, reciben la potencia de un conjunto motor-reductor.
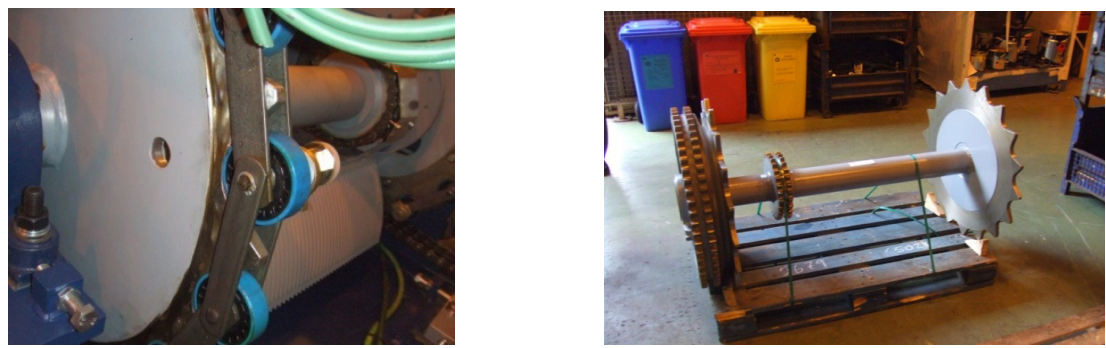

Gráfico 6. Sistema de tracción de una escalera mecánica convencional.

\section{MODELO DE SIMULACIÓN DINÁMICA}

El modelo de simulación de la escalera tiene varios apartados según los objetos simulados.

\subsection{CUERPOS}

Estos definen los objetos reales tridimensionales y recogen las características geométricas, dimensionales e inerciales. Los cuerpos incluidos en la metodología son las guías, interiores y exteriores, incluyendo las de la cabeza tensora, los eslabones, los rodillos y los peldaños.

\subsection{JUNTAS}

Estas definen las relaciones y restricciones de movimiento entre los cuerpos. El programa de simulación SIMPACK asocia una junta a cada cuerpo generado en el modelo. Cada junta define los grados de libertad existentes entre dos marcadores. Un marcador es un sistema de referencia, fijo o móvil, a asociado a un objeto. Una junta tiene un marcador from, perteneciente a otro cuerpo 


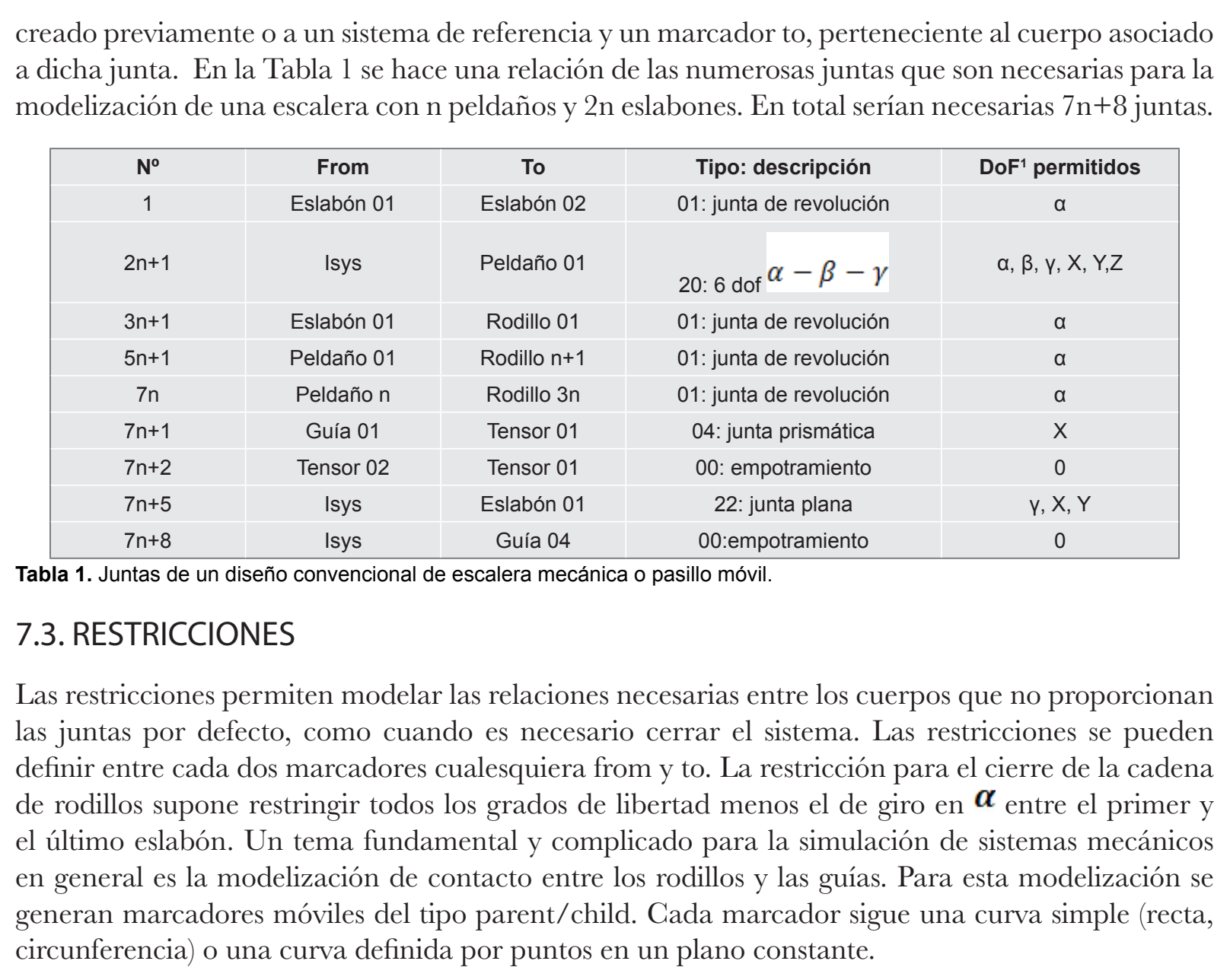




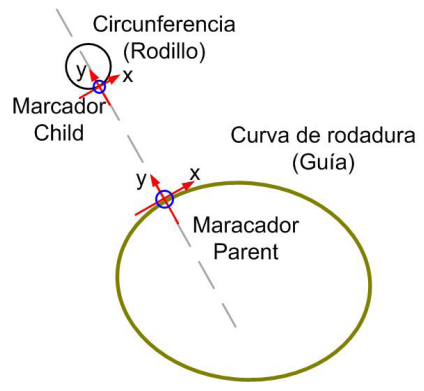

\begin{tabular}{|c|c|c|c|c|}
\hline No & From & To & Tipo: descripción & DoF restringidos \\
\hline 1 & Rodillo 01 & Guía 01 & 90: entre marcadores móviles & Y, X \\
\hline $4 n-3$ & Rodillo $4 n-3$ & Guía 01 & 90: entre marcadores móviles & Y, X \\
\hline $8 n-1$ & Rodillo $4 n-1$ & Tensor 03 & 90: entre marcadores móviles & Y, X \\
\hline $8 n+1$ & Eslabón 01 & Eslabón $n$ & 25: definida por el usuario & X, Y \\
\hline $8 n+2$ & Eslabón 02 & Eslabón $2 n$ & 25: definida por el usuario & X. $\mathrm{Y}$ \\
\hline
\end{tabular}

Tabla 2. Restricciones de un diseño convencional de escalera mecánica o pasillo móvil.

\subsection{FUERZAS APLICADAS}

Son las que representan las acciones reales que deberían de existir en un modelo de escalera mecánica. Estas fuerzas pueden dividirse en varios subsistemas. Las primeras para el accionamiento, que sujeta los peldaños a las dos cadenas de rodillos mediante muelles tridimensionales, incluyendo valores muelles-amortiguadores lineales y de torsión, y que suponen $2 \mathrm{n}$. Las siguientes son para la modelización del contacto entre los rodillos y las guías, siendo un total de 8n. El sistema de tracción es lineal y aplica una fuerza en cada articulación entre eslabones en el sentido tangencial a la guía en la zona de control empleada. Estas fuerzas suponen un número de 2n. La última fuerza es de la estación tensora se modeliza con un solo valor y precarga. En total hay que introducir $12 n+1$ fuerzas en el modelo 


\begin{tabular}{|c|c|c|c|c|}
\hline $\mathbf{N}^{\circ}$ & From & To & Tipo: descripción & Dirección \\
\hline 1 & Eslabón 01 & Peldaño 01 & 05: Muelle-Amortiguador en paralelo & $\alpha, \beta, \gamma, X, Y, Z$ \\
\hline $2 n+1$ & Guía 01 & Rodillo 01 & “ & $\mathrm{X}, \mathrm{Y}$ \\
\hline $6 n$ & Guía 04 & Rodillo 4n & “ & $X, Y$ \\
\hline $6 n+1$ & Tensor 01 & Rodillo 01 & “ & $X, Y$ \\
\hline $10 n+1$ & Isys & Rodillo 01 & “ & $\mathrm{X}$ \\
\hline $12 n+1$ & Isys & Tensor 01 & $\begin{array}{c}\text { 05: Precarga }+ \text { Muelle-Amortiguador en } \\
\text { paralelo }\end{array}$ & $x$ \\
\hline
\end{tabular}

Tabla 3. Fuerzas aplicadas en un diseño convencional de escalera mecánica o pasillo móvil.

\subsection{SISTEMA TENSOR}

Un problema particularmente difícil de resolver en simulación es el de las guías de geometría variable. Los programas de simulación se limitan simplemente a no aceptarlas. Si se modeliza una geometría particular para el tensor, diferente de la guía, aparecen posiciones singulares que falsean la modelización del contacto entre tensor y rodillos.
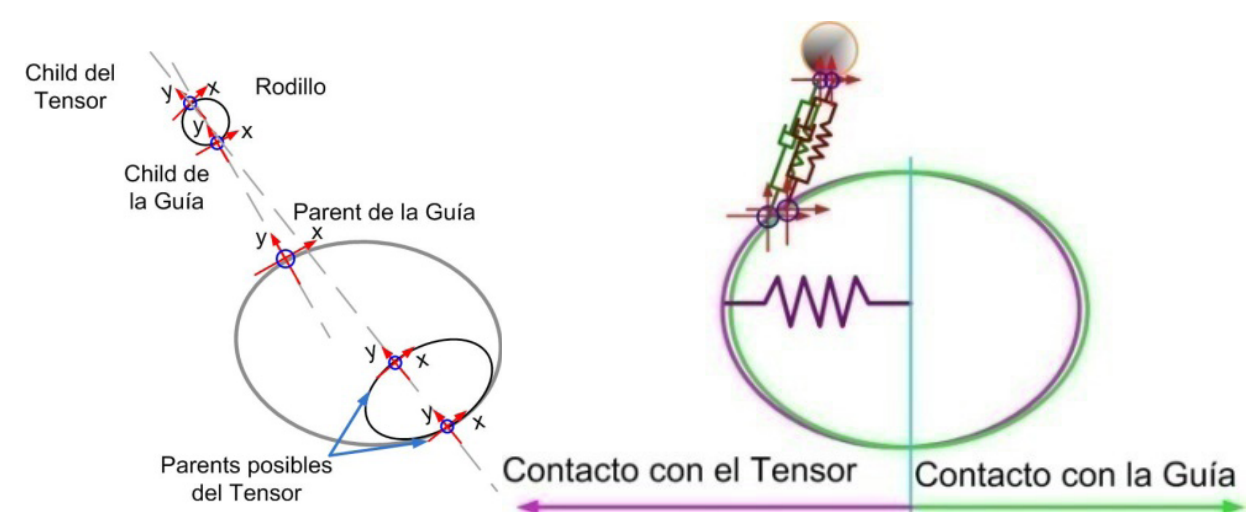

Gráfico 7. Posición singular de dos marcadores parent/child Diseño del sistema tensor.

En este trabajo se ha obtenido la solución aplicando los principios inventivos de TRIZ y consiste en definir una guía del tensor geométricamente igual a la de toda la guía. A esta geometría del tensor se le impone una junta prismática respecto al sistema de referencia fijo, Isys. Se definirán dos fuerzas de contacto entre cada par de marcadores móviles mediante expresiones que detecten en qué zona se encuentra cada rodillo. Así, la expresión que defina la fuerza de contacto entre el tensor y un rodillo concreto, se anulará cuando ese contacto se dé con la guía fija. 
La existencia de contraguías se puede introducir teniendo en cuenta una holgura en la expresión del muelle-amortiguador bidireccional. De esta forma la reacción que recibe un rodillo a lo largo de un ciclo es la suma de ambas fuerzas, las debidas a la zona de guía y la zona de tensor. Estas fuerzas están representadas en el Gráfico 8, observándose una suave transición entre ambas zonas. En la zona de transición, de longitud no nula, se reparten la reacción entre el tensor y la guía.

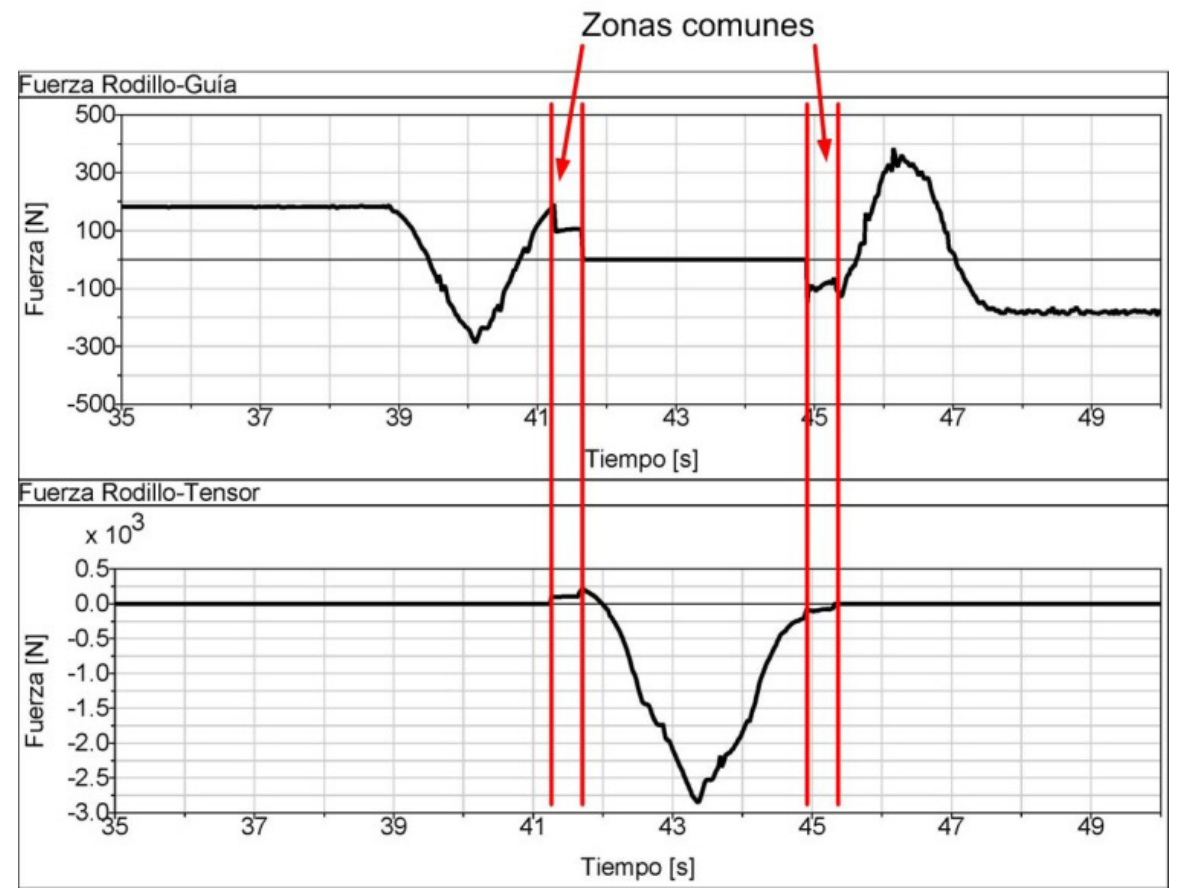

Gráfico 8. Dos fuentes de reacción del rodillo: guía (arriba) y tensor (abajo).

\subsection{SISTEMA DE TRACCIÓN}

Una primera opción para la modelización del sistema de tracción es emplear el módulo CHAIN del software SIMPACK que permite la modelización de cadenas. Este es el caso de algunos de los modelos desarrollados en [9]-[11]. Sin embargo, la especialización de este módulo supone también falta de flexibilidad. Para superarla se emplea el SIMPACK básico, y se describirá un sistema de tracción basado en un sistema de control, metodología que permite modelos más genéricos. 
Para modelizar el sistema de control es necesario primeramente seleccionar la zona de control, que es la cabecera superior en una escalera convencional y una zona recta en la parte superior de la escalera modelizada. La variable de control es la velocidad lineal de la cadena que es comparada con la velocidad de consigna. Mediante un control proporcional a esta comparación se aplica una fuerza a través de un actuador.

Ecuación 1.Fuerza de tracción aplicada.

$$
F=k p *(\text { Vreal }- \text { Vref })
$$

La fuerza aplicada debe ser tangencial a la guía. De esta forma basta que sea aplicada siguiendo la dirección del eje X de cualquiera de los marcadores parent/child definidos entre las curvas del rodillo y de la guía.
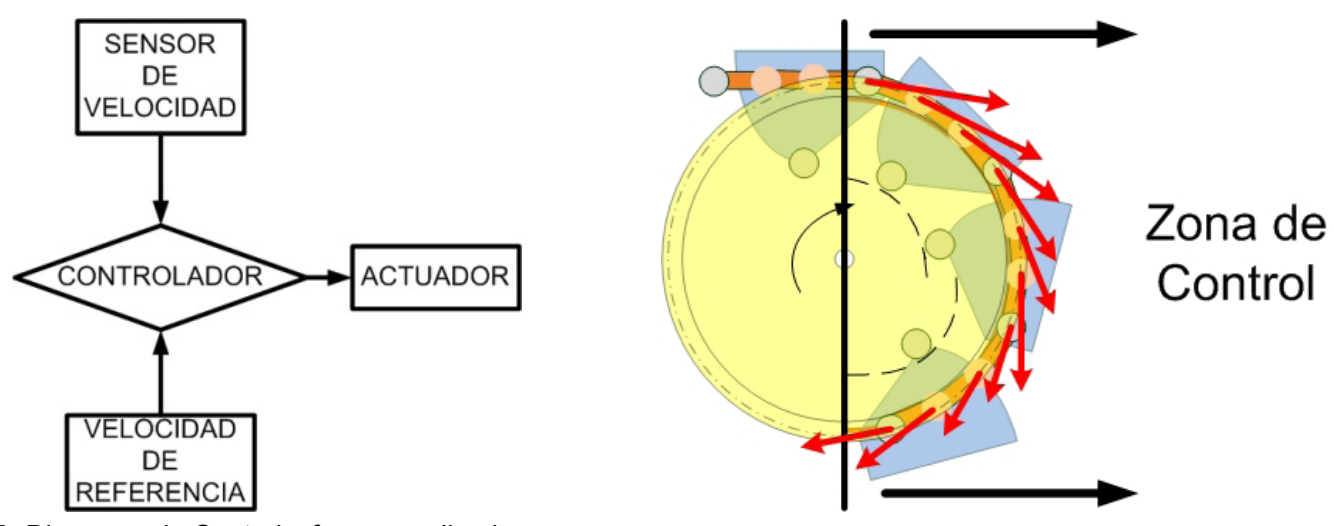

Gráfico 9. Diagrama de Control y fuerzas aplicadas.

\section{GENERACIÓN PARAMÉTRICA ESCALABLE DEL MODELO}

Estos modelos aquí descritos son resueltos convenientemente por el programa de simulación siempre y cuando se hayan introducido correctamente los parámetros de los elementos y sus posiciones iniciales. Esto es una tarea abrumadora y muy expuesta a errores. Para el modelo base de este estudio, que consta de $n=60$ peldaños y cadenas con un eslabón por peldaño, el número de cuerpos a simular es de unos 200, las restricciones cerca de 500 y las fuerzas cerca de 700 . En total cerca 
de 1.400 elementos con una media de 3 valores por elemento nos acerca a más de 4.000 valores a introducir. La escalera modelada corresponde a un desnivel de 4,5m. Hay escaleras que superan ampliamente los 20m y tienen cadenas de hasta 3 eslabones por peldaño.

Cualquier error en esto valores se traduce en errores de simulación o en la imposibilidad de esta por falta de convergencia en el estado inicial [12]. Adicionalmente está el problema de las geometrías de las guías que necesitan ser precisas, varían con cualquier cambio de configuración y deben estar en un formato que acepte el programa de simulación.

Los hechos relatados aquí justifican la escala literatura de simulación de escaleras existente.

Es una parte fundamental de este trabajo el desarrollo de todas las herramientas de automatización que permiten la introducción de un modelo escalable en función de unos pocos parámetros a introducir manualmente.

La automatización y escalabilidad afecta no sólo a la introducción del modelo, sino también a varios procesos más. El primero a la simulación en sí, con varias simulaciones sucesivas con diferentes condiciones de funcionamiento. Otro proceso es la selección, tratamiento y presentación de la miríada de resultados que produce la simulación, para que se convierta en información útil para analizar la dinámica de la escalera. El tratamiento de los datos de instrumentación es un proceso con entidad propia pero íntimamente ligado a los de simulación.

Entre las herramientas desarrolladas se ha considerado imprescindible las que permiten correlacionar los resultados experimentales con los de simulación para la mejora en la estimación de los valores de parámetros desconocidos o de difícil medición.

\section{MODELO REAL INSTRUMENTADO VALIDACIÓN}

El prototipo de escalera mecánica que se instrumentó está descrito con más detalle en otras publicaciones [12]. Se emplearon acelerómetros tridimensionales para medir las aceleraciones en los peldaños, galgas extensiométricas para medir la tensión en cada malla de las cadenas, galgas extensiométricas para medir la reacción en partes curvas de la guía y sensores laser para medir el desplazamiento de la estación tensora. 
Dado que los peldaños y las cadenas están en movimiento continuo fue necesario adaptar equipos portátiles que estuvieran embarcados en los peldaños y registraran aceleraciones y tensiones.

Todos los dispositivos de medida estaban al menos duplicados para evitar la pérdida de información o mejorar la precisión de la medida.

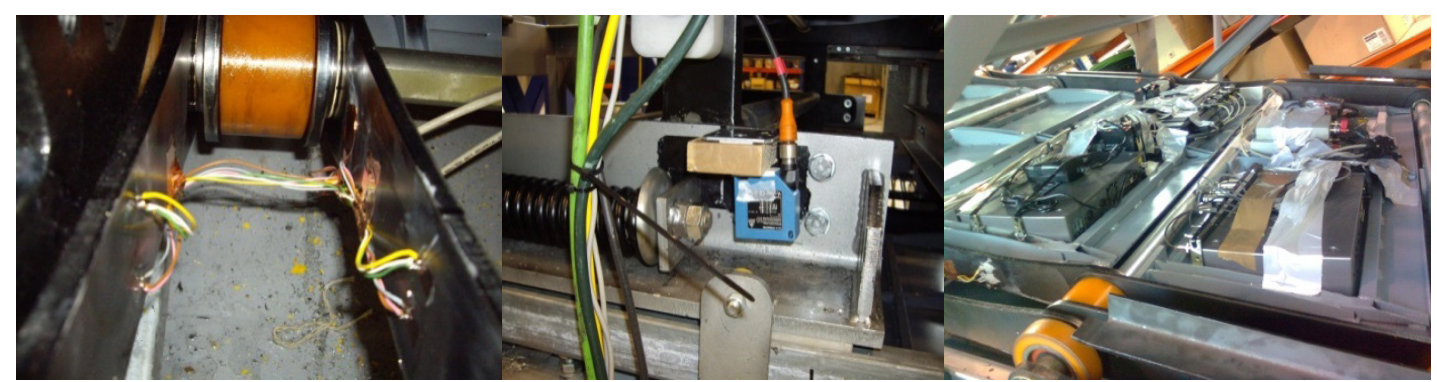

Gráfico 11. Sensores en un prototipo real: galgas, sensor laser, acelerómetros y equipo de registro.

Se hizo funcionar el prototipo siguiendo un plan de variación de condiciones donde se cambiaba la velocidad nominal, el sentido de giro, la precarga del tensor y la aceleración de arranque y parada. Cada escenario de funcionamiento se repetía al menos dos veces. La escalera funcionaba sin pasajeros al no disponer de un utillaje adecuado que hiciera el embarque y desembarque adecuadamente.

\section{RESULTADOS Y DISCUSIÓN}

La aceleración del peldaño que marca la seguridad y confort del pasajero durante todo el trayecto y es un marcador fundamental para la existencia de ruidos por vibración y sobrecargas por posibles resonancias.

Los valores dinámicos fundamentales que interesan para la evaluación de un diseño son en primer lugar la tensión de la cadena que determina la duración de sus articulaciones, la fuerza motora a aplicar, la robustez de la estructura portante y parte del desgaste de los rodillos. La reacción de los rodillos sobre las guías marca la duración de las bandas de rodadura de los mismos y la robustez de las guías para no sufrir deformaciones inadmisibles. La aceleración del peldaño que marca la seguridad y confort del pasajero durante todo el trayecto y es un marcador fundamental para la 
existencia de ruidos por vibración y sobrecargas por posibles resonancias. El desplazamiento del tensor es un movimiento oscilatorio que tiene gran influencia en el comportamiento.

Tras el arduo procesamiento de los datos de instrumentación, se sincronizaron con los datos de simulación, tanto para el punto inicial de ciclo como para la velocidad media. Con los datos sincronizados se estimaron los parámetros de simulación en función de la correlación de los datos simulados con los datos experimentales para obtener los parámetros más cercanos a la realidad.

Esta primera gráfica muestra conjuntamente la tensión de la cadena medida y la simulada. Lo primero a destacar es la buena correlación general existente entre las señales medidas y simuladas. Los picos se producen simultáneamente en una curva y otra, las zonas más estacionarias se solapan en gran medida y también las oscilaciones de 0,8 segundos de periodo que es la frecuencia de paso de peldaños y escalones.

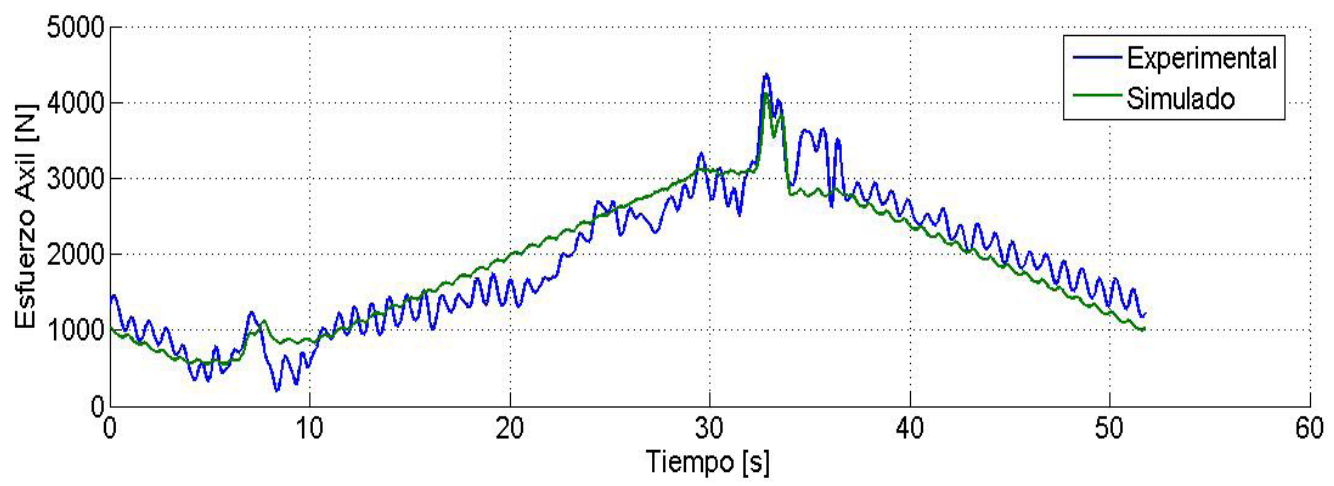

Gráfico 11. Tensión de un eslabón: filtro paso bajo de $2 \mathrm{~Hz}$.

También se pueden ver las diferencias. Las oscilaciones de tensión experimentales resultas de mayor amplitud que las simuladas. La gráfica experimental tiene más picos y es menos regular que la simulada. Evidentemente en un modelo tan complejo no se puede exigir la superposición exacta pero si estudiar el origen de las discrepancias. El origen puede ser errores de medida, falta de precisión o linealidad, ruidos, defectos de tratamiento de datos, o bien defectos de construcción del prototipo que introduzcan perturbaciones en la regularidad de funcionamiento. Pero también las discrepancias se pueden deber al modelo, bien en el valor de sus parámetros o bien en su estructura. El valor de los parámetros se ha optimizado con los experimentales pero siempre estarán sujetos 
a incertidumbre. Respecto a la estructura, es difícil que incluya los defectos de fabricación, salvo que se introduzcan de forma estadística. Son los defectos de estructura los que interesan para un refinamiento del modelo pero exigen un estudio local más profundo.

La siguiente gráfica se refiere a la aceleración de un peldaño. Las conclusiones son similares a la gráfica anterior, buena correlación general, picos experimentales más altos que los simulados.

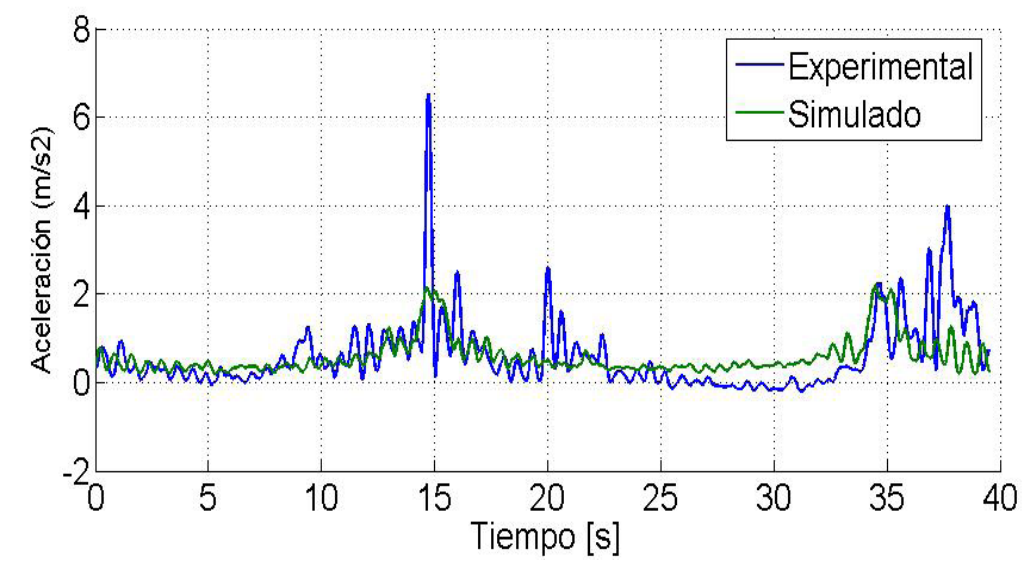

Gráfico 12. Aceleración de un peldaño: filtro paso bajo de $0.2 \mathrm{~Hz}$.

La pregunta es si estos comportamientos más bruscos se deben a deficiencias del prototipo, holguras, desalineaciones, o bien a simplificaciones del modelo que debe refinarse más.

En esta última gráfica de resultados se muestra el desplazamiento del tensor con una escala temporal ampliada. La superposición es casi perfecta salvo por algunos picos experimentales. 


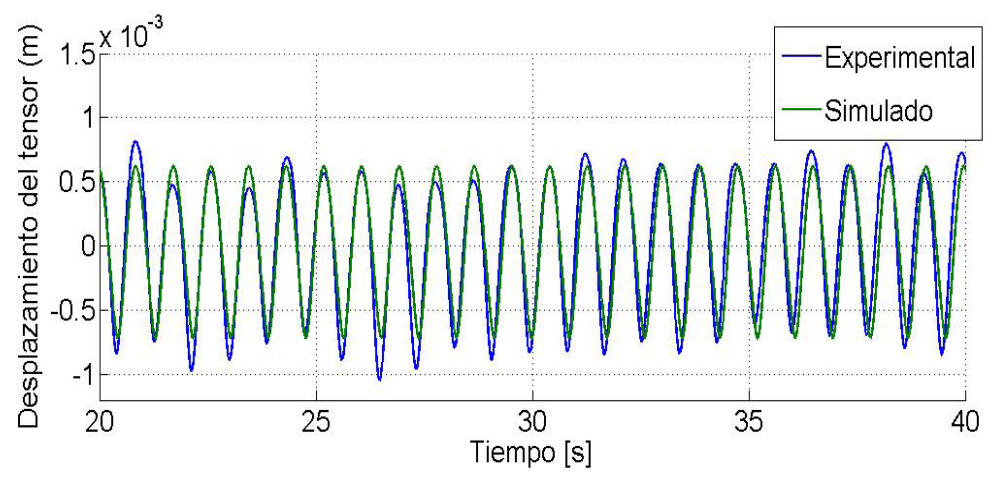

Gráfico 13. Desplazamiento del tensor.

El tensor es el subsistema más sencillo y por tanto el que mejor representa el modelo de simulación.

Se podría concluir que lo más probable es que las diferencias de regularidad sean por defectos del prototipo y las diferencias en amplitudes por simplificaciones del modelo.

Los valores estáticos de tensión del modelo y los que se pueden inferir de los valores medios experimentales, coinciden con los cálculos clásicos estáticos que se pueden hacer por medios más sencillos, lo que redunda en la validez del modelo y en la importancia de refinarlo.

\section{CONCLUSIONES}

Si no se evoluciona se extingue. Esto se podrá aplicar algunos fabricantes de escaleras mecánicas. Forzar esta evolución requiere contar con herramientas que permitan la verificación de las ideas creativas que se vayan produciendo.

En este trabajo se han descrito las herramientas que permiten valorar la calidad de un diseño existente o el valor añadido de una mejora entre dos diseños distintos.

La alta correlación entre los modelos real y simulado, avalan el uso de esta metodología y también indican el camino para ir refinando y ampliando a esta. 


\section{REFERENCIAS BIBLIOGRÁFICAS}

[1] Cano-Moreno, J. D. y Cabanellas Becerra, J. M. (2017). Evolución de las escaleras mecánicas: divergencia entre los diseños teórico y real. DYNA, 92(3), 264-268.

[2] S. GmbH. (2018). Multi-Body Simulation. SIMPACK MBS Software. [En línea]. Disponible en: http://www.simpack.com/. [Accedido: 01-jun-2018].

[3] Klisch, T. (1999). Contact mechanics in multibody systems. Mechanism and Machine Theory, 34(5), 665-675.

[4] Kwon, Y. S. (1999). Dynamic Analysis Step-By-Step, Elevator World, 47(1), 32.

[5] Kwon, Y.-S., Copeland, G. S. y Park, N.-G. (2005). A Multibody Dynamic Model for Escalator Handrail Systems and its Application to Dynamic Characteristics, Multibody Syst Dyn, 13(2), 253-266.

[6] Isotani, H., et al. (2002). Technology for Developing Escalator Mechanisms Using Multibody Dynamics Analysis. En: The Proceedings of the Elevator, Escalator and Amusement Rides Conference. (4952).

[7] Cabanellas Becerra, J. M., et al. (2010). Escalera mecánica. España: ES2319082B1.

[8] Cabanellas Becerra, J. M., et al. (2011). Sistema de accionamiento de una cadena de arrastre. España: ES2351000B1.

[9] Cano-Moreno, J. D., et al. (2009). Methodology for Flexible Modelling of Escalator Multibody Systems. En: Proceedings Industrial Simulation Conference. (227-231). UK: Loughborough. (227-231).

[10]Cabanellas Becerra, J. M., et al. (2008). Advanced Dynamical Models for Escalators Simulation, Elevator World, 56(11), 136-151.

[11]Cabanellas Becerra, J. M., et al. (2008). Mejora de un diseño de más de 100 años. Nuevos conceptos en escaleras mecánicas. En: XVII Congreso Nacional de Ingeniería Mecánica. España: Gijón. ISBN 0212-5072.

[12] Cano-Moreno, J. D. (2012). Metodologías para la Modelización, Validación y Diseño Innovador de los Sistemas Multicuerpo del Transporte Vertical de Personas. Tesis (Doctoral). Universidad Politécnica de Madrid: E.T.S.I. Industriales. 


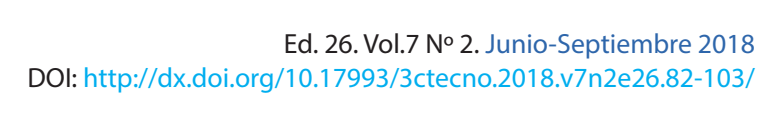

| 103 